Americans are at this moment removing 'Pamaquine' and chaulmoogra oil from their Pharmacopœia. They do not include leptazol, or nikethamide, or lobeline, and seem to rely on picrotoxin as the only official analeptic. They include no acridine antiseptic.

Another difficulty is the question of nomenclature. New pharmacopœias generally contain a few new names for old drugs and omit the old names, which are unsuitable for official use for one reason or another. Sometimes these new names explain themselves; benzalkonium is fairly obviously a cationic detergent. Sometimes their interpretation forms a test of general knowledge. Iodopyracet is the new American name for diodone, which is used to display the renal pelvis under X-rays. Some readers will no doubt be driven to other works of reference to confirm the fact that these two substances are, in fact, identical.

Some attempt is made to achieve agreement about such neologisms between the British and the Americans, but the results are disappointing. The Americans use ' $f$ ' where the British use 'ph', and simplify spelling in various other ways. The drugs known in America as epinephrine, menadione, tetracaine, quinacrine, and anhydrohydroxyprogesterone are known in Britain as adrenaline, menaphthone, amethocaine, mepacrine and ethisterone respectively.

The new work contains many admirable features. The official names have been chosen so as to bring allied preparations together in the alphabetical arrangement ; for example, tincture of opium is called opium tincture, so that it comes near opium itself. Metric doses have precedence over avoirdupois ; this convention will be welcomed internationally. English names have precedence over Latin names; this convention will be welcomed in English-speaking countries. There is much incidental information about the properties of a wide range of substances which are only occasionally used by pharmacists as reagents, and about the measurement of viscosity, $p \mathrm{H}$, turbidity, melting points, optical relation, etc.

Methods of biological assay are described for such substances as epinephrine, posterior pituitary, insulin, vitamins $A, B$ and $D$, and all preparations containing cardiac glycosides, and there are microbiological methods for nicotinamide and riboflavin. Such biological methods are not applied to pure active principles such as epinephrine or ouabain, but they are applied to preparations of these active principles, such as epinephrine solution or ouabain injection. In these cases, the assay is clearly not intended to be applied to each sample, but only used in cases of doubt or dispute. In the case of other drugs, such as insulin and digitalis tincture, the assay forms an integral part of the manufacture, and the exact procedure is prescribed in great detail, which leaves much less to the discretion of the individual than is the custom in Britain. Some of the details of the techniques will no doubt be criticized. Cats are to be used for the assay of digitalis, and methods using frogs are not recognized. There is no convincing evidence to justify this decision. Rabbits, and not mice, are to be used for the assay of insulin, and they must receive three doses of the unknown preparation and one dose of the standard, instead of two doses of each, as is customary here. The attempt to make these tests foolproof has made them too rigid.

The assays of various vitamins are not designed to measure their concentration, but only to ensure a certain minimum activity. This decision greatly simplifies the interpretation of the result. The standard preparations to be used for these assays are distributed officially; the Pharmacopœeia seems to contain no information about their composition. It would be interesting to have been told whether the standard for vitamin $D$ in cod liver oil is vitamin $D_{3}$, as it ideally should be, or calciferol.

For various reasons, some of which are mentioned above, this Pharmacopœia could not be officially adopted as it stands in Great Britain, but it contains much that we can admire, and might copy. The Americans are to be congratulated on a fine piece of work.

J. H. GADDUM

\section{METHODS IN CELLULOSE CHEMISTRY}

The Methods of Cellulose Chemistry

Including Methods for the Investigation of Substances associated with Cellulose in Plant Tissues. By Dr. Charles Dorée. Second edition, revised and enlarged. Pp. xii +543. (London: Chapman and Hall, Ltd., 1947). 42s. net.

7 HE earlier edition of this book has been of very 1 great value to all whose interests have been in the field of cellulose chemistry, and there can be no doubt, therefore, of the welcome which will be accorded to a second edition. The full usefulness of the volume can be appreciated only when the complete title is considered, since the reader will find, in addition to the multifarious methods of investigation applicable to cellulose and its derivatives, concise accounts of the methods of attack which have been developed for investigations on lignin, hemi-celluloses and pectic materials.

Much has been added to our knowledge of all these substances in the course of the fifteen years which have elapsed since the first edition was published, and Dr. Dorée has now undertaken with conspicuous success the formidable task of incorporating new. matter and revising the old. No fundamental changes have been found necessary, but a great deal of new material now finds its place in the book. In Part I methods used in the examination of normal cellulose are collected, including the measurement of physical properties, methods of analysis applicable to cellulose, mercerized cotton, rayons, hydrocelluloses and oxycelluloses. There is a chapter devoted to the investigation of damage in cotton and linen fabrics. The second part is concerned with synthetic derivatives of cellulose, including esters and ethers, and on this topic much new matter has been added in the present edition. The third part deals with the compound celluloses, and in addition to a chapter on the qualitative and quantitative examination of plant tissues there are detailed accounts of procedures for the estimation of cellulose and lignin, and of the standard methods for the estimation of furfural, uronic acid and methoxyl. Chapters are included also on the analysis of wood, and on the chemistry of lignin and the hemi-celluloses.

The book is designed for use by the practical worker in the laboratory. It is well illustrated with figures and graphs and is fully documented. Precise and detailed directions are given for all the analytical procedures. The comprehensive author- and subjectindexes facilitate reference, and the book has been excellently produced in a clear type which is a pleasure to read. Dr. Dorée is to be warmly congratulated on the appearance of this second edition of a most useful and valuable work.
E. L. HrRst 